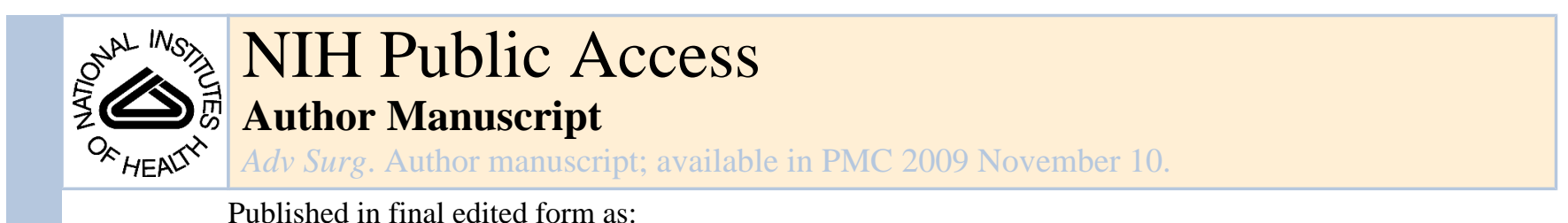

Published in final edited form as:

Adv Surg. 2008 ; 42: 249-260. doi:10.1016/j.yasu.2008.03.002.

\title{
Gene Expression Profiling of Breast Cancer
}

\author{
Ting Bao and Nancy E. Davidson
}

\section{Introduction}

Modern technology has brought breast cancer research and clinical care to a new era. In the past, breast cancer diagnosis, prognosis, and treatment decisions were based on clinicalpathological analysis of the breast cancer tissue and axillary lymph nodes. Breast cancer was classified according to tumor type (ductal vs. lobular infiltrating carcinoma), histological grade (I to III), steroid hormone receptor and HER2 status (positive vs negative), and cancer involvement of the lymph node(s) and distant organ(s). Forecasts about prognosis and recommendations about treatment would be based on those features, which imperfectly predict clinical outcomes and could result in excessive treatment of many patients with chemotherapy for marginal benefit. Hence, new methods are needed to further our understanding of breast cancer in order to optimize and individualize breast cancer treatment. Gene expression profiling has been intensively studied by breast cancer researchers and results of these studies have begun to be embraced by clinical oncologists in their day-to-day practice. Thus it is important to understand the technique itself and its potential applications.

The development of gene microarray techniques has enabled scientists to detect the differences of gene expression among thousands of genes simultaneously and thus create gene expression profiles for different types of breast cancer. ${ }^{1}$ Gene expression profiling of breast cancer has improved our understanding of the heterogeneity of breast cancer on the genomic level; challenged the current classification of breast cancer; served as an important prognostic indicator; and most importantly, begun to guide our treatment in women with early breast cancer. This chapter will provide a basic description of gene expression profiling and an overview of the three major potential clinical applications of this technique.

\section{Gene Expression Profiling}

Gene expression profiling is a genetic microarray analysis of genetic transcriptional variations between normal and malignant cells. It details the expression levels of thousands of genes in breast cancer and draws molecular portraits of breast cancer. Gene microarray analysis was first described in mid 1990s, ${ }^{2,3}$ and was developed based on the belief that only a fraction of the genes are transcribed into messenger RNAs (mRNA), and subsequently translated into proteins, ultimately resulting in different characteristics and functions of the cells. Gene expression profiling involves using complementary DNA (cDNA) or oligonucleotide microarrays to probe gene expression in the form of messenger RNA (mRNA) that are extracted from fresh frozen tissue ${ }^{4}$ or cDNA obtained as reverse transcriptase-polymerase chain reaction (RT-PCR) products from mRNA extracted from paraffin-fixed tissue. ${ }^{5}$ During the discover

(C) 2008 Mosby, Inc. All rights reserved.

Nancy Davidson, M.D., Department of Oncology, The Johns Hopkins Hospital, 600 N. Wolfe Street, Baltimore, MD 21287, davidna@jhmi.edu.

Publisher's Disclaimer: This is a PDF file of an unedited manuscript that has been accepted for publication. As a service to our customers we are providing this early version of the manuscript. The manuscript will undergo copyediting, typesetting, and review of the resulting proof before it is published in its final citable form. Please note that during the production process errors may be discovered which could affect the content, and all legal disclaimers that apply to the journal pertain. 
process expression data are analyzed using either unsupervised or supervised analyses. The unsupervised analysis disregards biological outcomes and seeks to detect the inherent differences and relationships among tissues through gene expression data alone. In contrast, the supervised analysis incorporates the known biological outcomes such as survival or recurrence to develop prognostic models.

\section{Applications}

\section{Use of gene profiles to sub-classify breast cancer}

Although the inherent variability of breast cancers has been recognized for decades, gene expression profiling has demonstrated the heterogeneity of breast cancer on the genomic level. In 2000, Perou et al. first described six intrinsic subtypes of breast cancer using unsupervised analysis of gene expression profiles of 65 breast caner surgical tissues. ${ }^{6}$ A set of 456 genes was identified as being associated with the differences among those breast cancer tissues. Initially six molecular subgroups were identified, luminal A, B, and C, HER2+/ER-, basallike and normal breast-like groups. ${ }^{7}$ With time it became apparent that the luminal $\mathrm{C}$ subtype is seldom seen and it is not certain whether the normal breast-like group truly represents breast cancer tissue or was merely a sampling error of the benign breast tissue embedded in breast cancer tissue. The remaining four intrinsic subtypes of breast cancer, luminal A and B, HER2 +/ER- and basal-like, are reasonably widely accepted at this time.

Reassuringly, the intrinsic subtypes correlate with the known pathological characteristics of the breast cancers, especially estrogen receptor alpha (ER) status and immunohistological features. Luminal A and B subtypes express ER protein, whereas HER2+/ER- and basal-like subtypes are ER-negative. Subtyping breast cancer into luminal or basal-like groups also correlates with pathological staining. Luminal tissues stain positive for keratins 8 and 18, whereas basal-like tissues stain positive for keratins 5 and 17. The importance of the intrinsic gene subtyping set has been validated through its application to available datasets. ${ }^{8}$ It has been demonstrated that each subgroup has distinct clinical outcomes. Luminal A patients have the best overall survival and disease free survival while luminal B and HER2+/ER- patients have intermediate outcomes, and basal-like patients do the worst. ${ }^{9}$

\section{Use of gene profiles to estimate prognosis}

Even though sub-classification of breast cancer is very useful in deepening our understanding of breast cancer, our ultimate goal is to use this information to predict disease prognosis and patient outcomes and to select treatment for the patients. Gene expression profiling has been used to refine our ability to define prognosis, that is, the natural history of a woman with newly diagnosed early breast cancer. Before the advent of genetic profiling, standard prognostic factors such as stage and biochemical receptor analysis were used to estimate prognosis. Computerized algorithms synthesizing these data like the well validated clinical-pathological prognostic indicator, Adjuvant! Online, are used widely to predict prognosis and treatment response. Because such prognostic indicators are imperfect, much work has been directed toward utilization of genetic profiles to improve individualized counseling, especially in patients with node-negative breast cancer. With the advent of gene expression profiling, at least six additional well characterized prognostic indicators have been developed and some of them are moving into the clinical setting. These six profiles are termed intrinsic subtypes, 70-gene profile, 76-gene prognostic classifier, wound response, 21-gene recurrence score and two-gene ratio prognostic models. Among them, the first four can only be derived using RNA from fresh frozen tissues whereas the last two can be done on fixed archived tissue. A summary of these six classifiers is shown in Table 1. 
As mentioned above, the intrinsic subtype model classified breast cancer tissues into four main subtypes and predicted that luminal A patients did the best, followed by luminal B and HER2 +/ER- patients, and basal-like patients did the worst. The Amsterdam 70-gene profile was developed using supervised analysis of 78 frozen breast cancer tissues from patients younger than 55 years of age with node-negative breast cancer. The Amsterdam team compared gene expression profiles of 34 patients who developed distant metastasis with the gene expression profiles of the remaining 44 patients who did not and identified a 70 -gene set to predict clinical outcomes. ${ }^{10}$ This gene set was subsequently validated externally in two retrospective studies. One study used the gene set to predict outcomes in 295 patients younger than 53 years of age with either lymph node positive or negative disease. The 180 patients with poor prognostic signature tumors had statistically significantly shorter 10 year distant-metastasis-free and overall survival than the 115 patients with good prognostic signatures regardless of their lymph node status (Fig 1). ${ }^{11}$ The second retrospective study applied the 70 -gene prognostic signature in 302 patients younger than 60 years of age with node-negative tumors and suggested that the 70-gene prognostic signature was better than "Adjuvant! Online" in predicting distant metastasis-free survival and overall survival. ${ }^{12}$ The US Food and Drug Administration (FDA) approved its commercial use in the form of the Mammaprint ${ }^{\circledR}$ assay and its utility is under evaluation in clinical trials to determine role of systemic therapy in node-negative breast cancer patients.

The 21-gene recurrence score is currently the most widely used gene expression profile prognostic model in the US. It was developed through evaluation of 250 genes that could putatively correlate with breast cancer recurrence based on existing literature, database and experimental evidence to identify a panel of 21 genes including 16 cancer-related genes (including HER2, ER related genes and proliferation genes) and 5 reference genes. Their combined expression was then assessed in tumor tissue derived from women who had previously participated in adjuvant therapy trials with known outcomes. It was possible to derive a recurrence score reflecting prognosis for women with node-negative ER-positive breast cancer receiving tamoxifen. The recurrence score was reported on a scale from 0 to 100 , with low risk defined as score less than 18, high risk defined as score greater than 30 and intermediate risk reflecting a score of 18-30. A useful attribute of this assay is that it does not require frozen tissues and can be done on fixed tissue. It was validated through its application to 668 out of 2,617 tamoxifen treated-patient samples collected in the NSABP B14 trial which examined the benefit of tamoxifen in hormone-receptor-positive, node-negative breast cancer. In these women who received only tamoxifen, $6.8 \%$ of patients in the low recurrence score group had distant recurrence at 10 years as compared with $14.3 \%$ in the intermediate-risk group and $30.5 \%$ in the high-risk group. The difference in the low-risk and the high-risk group is statistically significant with $\mathrm{p}$ value $<0.001$. Multivariate analysis revealed that the predictive power of the 21-gene recurrence score was independent of age and tumor size. ${ }^{13}$ Another study demonstrated that the 21-gene set is more accurate in predicting outcomes than Adjuvant! Online. ${ }^{14}$ The predictive power of the 21 -gene recurrence score was further validated in predicting breast cancer related mortality, ${ }^{15}$ and responsiveness to chemotherapy and hormonal therapy (Fig 2). ${ }^{16,17}$ Based on the strong evidence of its prognostic and predictive power, the 21-gene recurrence score has also been cleared for commercial use in an assay known as OncotypeDx®.

Several other prognostic models have not yet fully made their way to clinical practice. The 76gene prognostic model was also developed to evaluate patients with node-negative breast cancer. Two hundred eighty-six such patients were divided into hormone-receptor-positive and negative groups before gene expression profiling was performed. A 76-gene prognostic signature was developed after comparing the two groups' gene expression profiles and has been used to predict disease free survival and overall survival in patients with node-negative early-stage breast cancer who have not received systemic therapy. ${ }^{18,19}$ The wound response 
gene expression profile came from an evaluation of the core serum response genes whose expression changes when fibroblasts are activated. ${ }^{20}$ It is believed that an active wound healing genetic profile predicts increased risk of metastasis and death in patients with breast, lung and gastric cancers. ${ }^{20}$ Finally the two-gene ratio model was developed in 60 ER-positive, earlystage breast cancer patients treated with tamoxifen. The ratio of the expression of homeobox 13 and interleukin 17B was used to predict disease-free survival in those patients, with the higher the ratio predicting worse the clinical outcome. It may be useful to identify estrogenreceptor-positive early-stage breast cancer patients who have a poor outcome with tamoxifen and could possibly benefit from additional therapy rather than tamoxifen ${ }^{21}$

It is interesting and sobering that there is very little overlap among genes identified in the above mentioned six major gene-expression prognostic indicators. It was therefore of considerable importance to understand if these prognostic indicators are concordant with each other. In 2006, the Perou group answered this key question through a retrospective concordance study by analysis of five of the gene-expression prognostic models-intrinsic subtype, 70-gene profile, wound response, recurrence score, and two-gene ratio-- on 295 breast cancer samples simultaneously. ${ }^{22}$ The study demonstrated that all but the two-gene-ratio model had a high degree of concordance with each other. The poor risk group was defined as basal-like, luminal B and HER2+/ER- intrinsic subtypes, poor 70-gene profile, high 21-gene recurrence score and activated wound response. All four models accurately predicted disease free survival and overall survival, correlated with histological grade, and provided additional prognostic power. The two most validated and clinically applied models, the 70-gene and 21-gene recurrence score, showed $81 \%$ agreement in outcome classification. Thus, even though there is marked difference in gene selection in the prognostic models, there is concordance among four out of five models, suggesting they identify a common set of biologic phenotypes contributing to similar estimates of prognosis. ${ }^{22}$

\section{Use of gene profiles to predict response to therapy}

Selection of therapy based on attributes of the tumor-The majority of newly diagnosed invasive breast cancers are early stage and hormone-receptor-positive and adjuvant endocrine therapy is viewed as standard. ${ }^{23}$ Current guidelines recommend consideration of adjuvant chemotherapy for these women if the tumor is larger than $1 \mathrm{~cm}$ or if the tumor is smaller than $1 \mathrm{~cm}$ with unfavorable histological features. ${ }^{24}$ It is estimated that some $80-85 \%$ of these women may be sufficiently treated with hormonal therapy alone. ${ }^{25}$ The key is to correctly identify the $15-20 \%$ patients with poor outcomes who will truly benefit from adjuvant chemotherapy. Clinical-pathological features have been used to predict chemosensitivity through decision aids such as "Adjuvant! Online," but many physicians and patients tend to err on the side of caution, which then results in many women being over-treated with chemotherapy. New biomarkers that would accurately guide treatment for the individual are needed.

The ultimate goal of gene expression profiling is to help the oncologist design an individualized treatment plan with maximum benefit and minimum toxicity through the use of predictive markers. Unlike the numerous prognostic indicators being developed as described above, there are very few models predictive for therapy that are available. Among them, the 21-gene recurrence score (OncotypeDx®, Genomic Health Inc, Redwood City, CA) is the most widely used genomic model to predict chemotherapy response in the US. The ability of the 21-gene recurrence score to predict chemotherapy response was validated in a retrospective analysis of the tissue blocks collected in the NSABP B20 trial, which tested the benefit of adding chemotherapy (CMF cyclophosphamide, methotrexate and 5-flurouracil or MF) to 5 years of tamoxifen in node-negative, ER- positive patients. Figure 2 illustrates the predictive value of the 21-gene recurrence score for the value of adding chemotherapy to tamoxifen. The study 
demonstrated that chemotherapy significantly decreased the absolute risk of distant recurrence rate at 10 years from $88 \%$ to $60 \%$ in patients with high recurrence scores (>30) and did not produce any substantial benefit in patients with low or intermediate recurrence scores. ${ }^{17}$ Based on this result in clinical practice some oncologists are using the 21-gene recurrence score in patients with node-negative, hormone-receptor-positive early-stage breast cancer to determine if patients would be candidate for chemotherapy in addition to hormonal therapy. The current consensus is that chemotherapy need not be offered if the recurrence score is less than 18 and should be offered if the recurrence score is higher than 30 . There is uncertainty about what to recommend for women with an intermediate recurrence score and a prospective randomized controlled trial termed Trial Assigning Individualized Options for Treatment (TAILORx) is ongoing in North America to answer this question.

TAILORx is the first clinical trial designed by the National Cancer Institute Program for the Assessment of Clinical Cancer Tests (PACCT), which aims to integrate modern molecular techniques into oncology practice to individualize cancer therapy. Its schema is shown in figure 3 . Among all the diagnostic and prognostic tests, the 21-gene recurrence score model was selected for initial testing because it is performed on fixed tissue rather than fresh frozen tissue, and its prognostic and predictive power has been extensively validated. ${ }^{26}$ This study was initiated to resolve the uncertainty about whether to offer chemotherapy to patients with intermediate recurrence scores who are potential chemotherapy candidates. Over ten thousand women with node-negative, hormone-receptor-positive, HER-2 negative breast cancer will be enrolled in this trial. Upon enrollment, a recurrence score will be obtained using the 21-gene/ OncotypDx assay on primary breast cancer tissue. Based on the score, participants will be assigned to three different treatment groups. Women whose score is less than 11 will receive hormonal therapy alone while those whose score is greater than 25 will receive chemotherapy followed by hormonal therapy. Those whose score is between 11 and 25 will be randomized to either chemohormonal therapy or hormonal therapy alone. This range was expanded from the original studies to minimize the potential for inadequate treatment of women whose scores fall in the intermediate and high risk zone. Disease free survival will be used as the primary end point of this study so that these results can be integrated with other trials of adjuvant therapy. When complete it is hoped that this trial will establish the use of the 21-gene assay to provide more individualized treatment to hormone-receptor-positive, early-stage breast cancer patients. It should also be noted that preliminary information suggests that this assay might also be useful in predicting chemotherapy benefit in women with node-positive, steroid receptor-positive breast cancer and this is also an area of active research.

A similar trial—-the Microarray in Node-Negative Disease may Avoid Chemotherapy (MINDACT) — study is taking place in Europe. MINDACT is a prospective, randomized study designed to compare the utility of the Amsterdam 70-gene signature (Mammaprint ${ }^{\circledR}$ ) with the established clinical-pathological criteria embodied in the "Adjuvant! Online" program to identify node-negative breast cancer patients for chemotherapy. It will also compare the efficacy and toxicity profiles of a docetaxel-capecitabine regimen and a standard anthracyclinebased regimen. Additionally, it will compare the efficacy of single agent hormonal therapy ( 7 years of the aromatase inhibitor, letrozole) with that of sequential endocrine therapy ( 2 years of tamoxifen followed by 5 years of letrozole). The MINDACT trial is a collaborative effort jointly conducted by a number of national and international clinical trial research organizations in Europe and the rest of the world, with the European Organization for Research and Treatment of Cancer (EORTC) as the coordinating center. In this study frozen breast cancer tissues removed at time of surgery from over 6,000 node-negative breast cancer patients will be evaluated by the 70-gene prognostic assay and conventional patient parameters will be assessed through "Adjuvant! Online." Patients who are felt to be high risk by both analyses will receive chemotherapy followed by hormonal therapy if they are hormone-receptor-positive. If both models predict patients to be low risk, they will receive hormonal therapy alone if it is clinically 
indicated. If, however, there is discordance between the two models, the patients will be randomized to chemotherapy plus hormonal therapy or hormonal therapy alone. Patients who are to received chemotherapy will be randomized to either a standard anthracycline-based regimen or docetaxel plus capecitabine. Hormonal therapy is offered to patients with hormonereceptor-positive breast cancer only who will be randomized to either seven years of letrozole or two years of tamoxifen followed by five years of letrozole (Fig 4). ${ }^{27}$

Selection of therapy based on attributes of the host-The future of breast cancer treatment is likely to be individualized therapy based on both tumor biology and host biology. There is increasing recognition that subtle changes in gene sequence, single nucleotide polymorphisms, may affect the ultimate function of the resulting product and that such variation may account for individual differences in efficacy and toxicity of treatment. In breast cancer, this area of pharmacogenetics is being explored as a possible determinant for response to tamoxifen. Many drugs are metabolized by CYP450 encoded enzymes. Within the family of CYP450, members like CYP2 and CYP3 are considered key drug metabolizers, whose enzyme activities are affected extensively by their genetic composition. Emerging research has suggested inherited genetic variation in the CYP2D6 gene may be associated with a reduction in concentration of the active metabolite of tamoxifen, endoxifene. This might in turn be associated with a poorer clinical benefit although the small studies completed to date have given mixed results on this question. ${ }^{28}$. A commercially available test, AmpliChip CYP450 test ${ }^{\circ}$ (Roche), provides comprehensive analysis of the CYP2D6 and CYP2C19 genes in a microarray-based assay. There is not a consensus about whether or how to use this assay at present because of uncertainty about whether individuals with variant CYP2D6 alleles would benefit from a change in therapy ${ }^{29}$ However, it is likely that these types of analyses, if properly derived and validated, will become a part of algorithms for selection and dosing of drugs in the future, both in cancer and other clinical settings.

\section{Conclusions and Future Directions}

Gene expression profiling is enabling scientists to understand the heterogeneous nature of breast cancer on a genomic level. Several gene expression profiles for breast cancers have emerged in the initial studies and appear to be generally concordant in their ability to predict poor outcome. Of these profiles, the OncotypeDx ${ }^{\circledR}$ and Mammaprint ${ }^{\circledR}$ assay are the best validated and are commercially available. Their role in clinical practice is being refined through ongoing clinical trials. Other efforts are directed at determining host factors that might help to identify prognosis as well as response and toxicity of therapy. It is expected that availability of these types of analyses will only increase in the future. It will be imperative that such assays be well validated and have implications for how to modify care for the individual patient.

\section{References}

1. van't Veer LJ, Paik S, Hayes DF. Gene expression profiling of breast cancer: a new tumor marker. J Clin Oncol 2005;23:1631-1635. [PubMed: 15755970]

2. Lipshutz RJ, Morris D, Chee M, et al. Using oligonucleotide probe arrays to access genetic diversity. Biotechniques 1995;19:442-447. [PubMed: 7495558]

3. Schena M, Shalon D, Davis RW, Brown PO. Quantitative monitoring of gene expression patterns with a complementary DNA microarray. Science 1995;270:467-470. [PubMed: 7569999]

4. Quackenbush J. Microarray analysis and tumor classification. N Engl J Med 2006;354:2463-2472. [PubMed: 16760446]

5. Cronin M, Pho M, Dutta D, et al. Measurement of gene expression in archival paraffin-embedded tissues: development and performance of a 92-gene reverse transcriptase-polymerase chain reaction assay. Am J Pathol 2004;164:35-42. [PubMed: 14695316] 
6. Perou CM, Sorlie T, Eisen MB, et al. Molecular portraits of human breast tumours. Nature 2000;406:747-752. [PubMed: 10963602]

7. Morris SR, Carey LA. Molecular profiling in breast cancer. Rev Endocr Metab Disord 2007;8:185198. [PubMed: 17464566]

8. Sorlie T, Tibshirani R, Parker J, et al. Repeated observation of breast tumor subtypes in independent gene expression data sets. Proc Natl Acad Sci U S A 2003;100:8418-8423. [PubMed: 12829800]

9. Sorlie T, Perou CM, Tibshirani R, et al. Gene expression patterns of breast carcinomas distinguish tumor subclasses with clinical implications. Proc Natl Acad Sci U S A 2001;98:10869-10874. [PubMed: 11553815]

10. van 't Veer LJ, Dai H, van de Vijver MJ, et al. Gene expression profiling predicts clinical outcome of breast cancer. Nature 2002;415:530-536. [PubMed: 11823860]

11. van de Vijver MJ, He YD, Van't Veer LJ, et al. A gene-expression signature as a predictor of survival in breast cancer. N Engl J Med 2002;347:1999-2009. [PubMed: 12490681]

12. Buyse M, Loi S, Van't Veer L, et al. Validation and clinical utility of a 70-gene prognostic signature for women with node-negative breast cancer. J Natl Cancer Inst 2006;98:1183-1192. [PubMed: 16954471]

13. Paik S, Shak S, Tang G, et al. A multigene assay to predict recurrence of tamoxifen-treated, nodenegative breast cancer. N Engl J Med 2004;351:2817-2826. [PubMed: 15591335]

14. Bryant, J. Oncotype DX correlates more closely with prognosis than Adjuvant Online; Ninth St Gallen International consensus Conference: Primary Therapy of early Breast Cancer; 2005.

15. Habel LA, Shak S, Jacobs MK, et al. A population-based study of tumor gene expression and risk of breast cancer death among lymph node-negative patients. Breast Cancer Res 2006;8:R25. [PubMed: 16737553]

16. Gianni L, Zambetti M, Clark K, et al. Gene expression profiles in paraffin-embedded core biopsy tissue predict response to chemotherapy in women with locally advanced breast cancer. J Clin Oncol 2005;23:7265-7277. [PubMed: 16145055]

17. Paik S, Tang G, Shak S, et al. Gene expression and benefit of chemotherapy in women with nodenegative, estrogen receptor-positive breast cancer. J Clin Oncol 2006;24:3726-3734. [PubMed: 16720680]

18. Foekens JA, Atkins D, Zhang Y, et al. Multicenter validation of a gene expression-based prognostic signature in lymph node-negative primary breast cancer. J Clin Oncol 2006;24:1665-1671. [PubMed: 16505412]

19. Wang Y, Klijn JG, Zhang Y, et al. Gene-expression profiles to predict distant metastasis of lymphnode-negative primary breast cancer. Lancet 2005;365:671-679. [PubMed: 15721472]

20. Chang HY, Sneddon JB, Alizadeh AA, et al. Gene expression signature of fibroblast serum response predicts human cancer progression: similarities between tumors and wounds. PLoS Biol 2004;2:E7. [PubMed: 14737219]

21. Ma XJ, Wang Z, Ryan PD, et al. A two-gene expression ratio predicts clinical outcome in breast cancer patients treated with tamoxifen. Cancer Cell 2004;5:607-616. [PubMed: 15193263]

22. Fan C, Oh DS, Wessels L, et al. Concordance among gene-expression-based predictors for breast cancer. N Engl J Med 2006;355:560-569. [PubMed: 16899776]

23. Li CI, Daling JR, Malone KE. Incidence of invasive breast cancer by hormone receptor status from 1992 to 1998. J Clin Oncol 2003;21:28-34. [PubMed: 12506166]

24. Carlson RW, Brown E, Burstein HJ, et al. NCCN Task Force Report: Adjuvant Therapy for Breast Cancer. J Natl Compr Canc Netw 2006;1:S1-S26.

25. Fisher B, Jeong JH, Dignam J, et al. Findings from recent National Surgical Adjuvant Breast and Bowel Project adjuvant studies in stage I breast cancer. J Natl Cancer Inst Monogr 2001:62-66. [PubMed: 11773294]

26. Sparano, J. Design of and Rationale for the Trial Assigning Individualized Options for Treatment (TAILORx). In: Govindan, R., editor. American Society of Clinical Oncology Educational Book. Vol. Vol. 43. Alexandria, VA: Greaves, Lisa; 2007. p. 34-39.

27. MINDACT Trial Outline. http://www.eortc.be/services/unit/mindact/documents/MINDACT_trial_outline.pdf 
28. Goetz MP, Rae JM, Suman VJ, et al. Pharmacogenetics of tamoxifen biotransformation is associated with clinical outcomes of efficacy and hot flashes. J Clin Oncol 2005;23:9312-9318. [PubMed: 16361630]

29. Stebbing J, Stearns V, Davidson NE. Role of CYP2D6 testing in selection of endocrine therapy for breast cancer. Pharmacogenomics 2007;8:1-3. [PubMed: 17187500] 


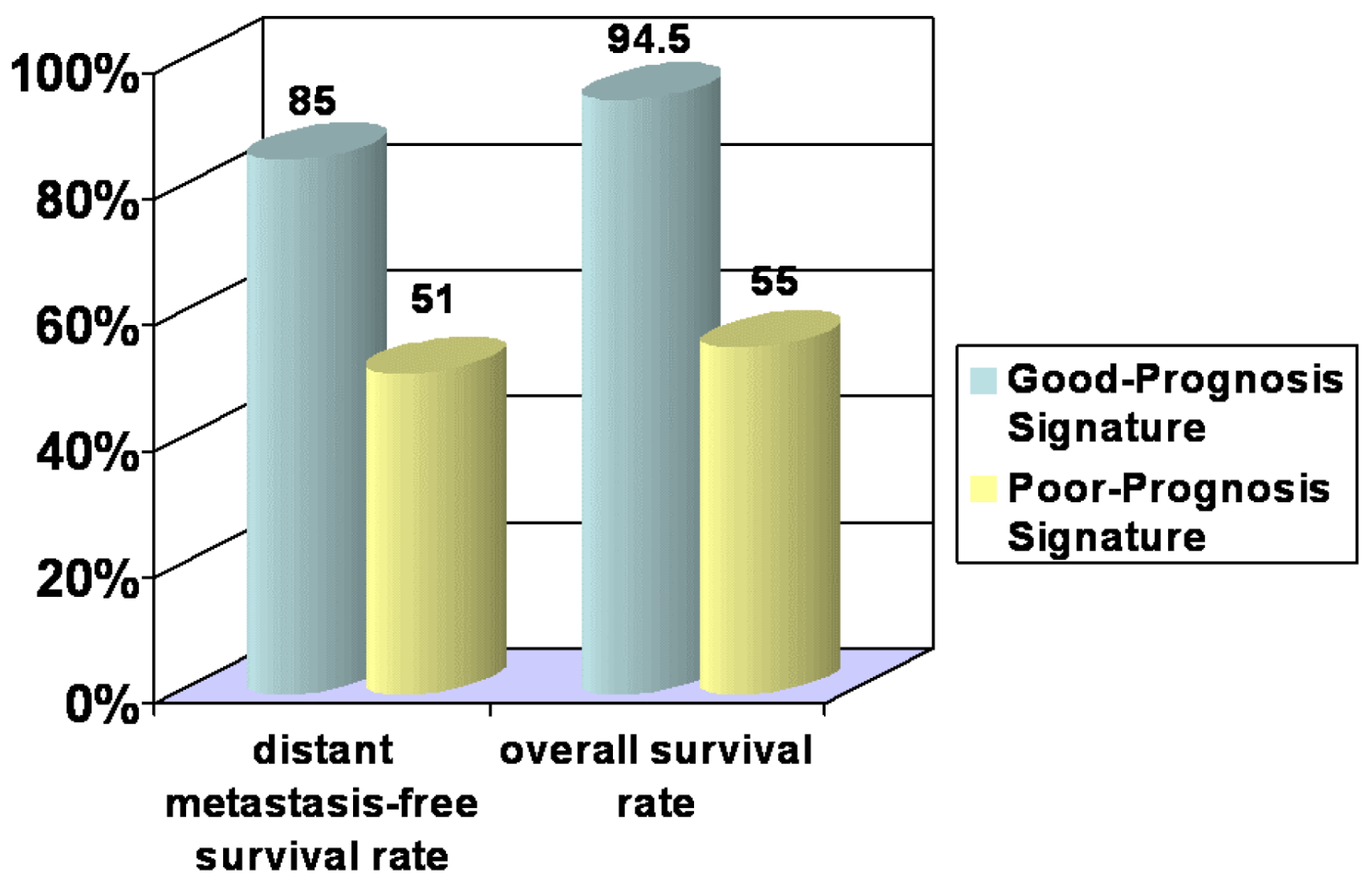

Figure 1.

Prognostic Value of 70-Gene Prognostic Signature (Based on data from van de Vijver, $2002^{11}$ ) 


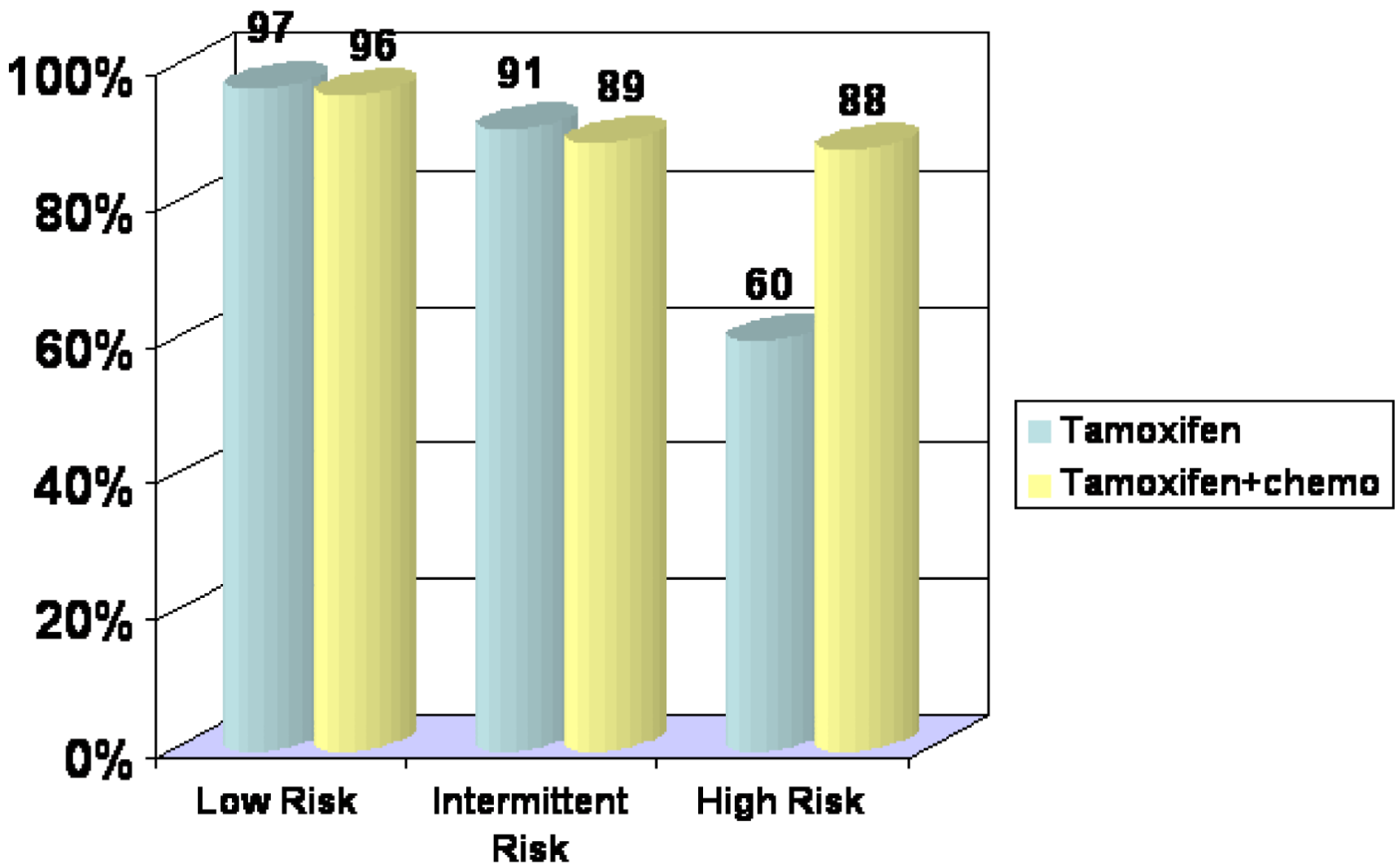

Figure 2.

Predictive Value of 21-Gene Recurrence Score for Addition of Chemotherapy (Based on data from Paik, 2006 ${ }^{17}$ )

Abbreviation: DRFS = distant recurrence-free survival 


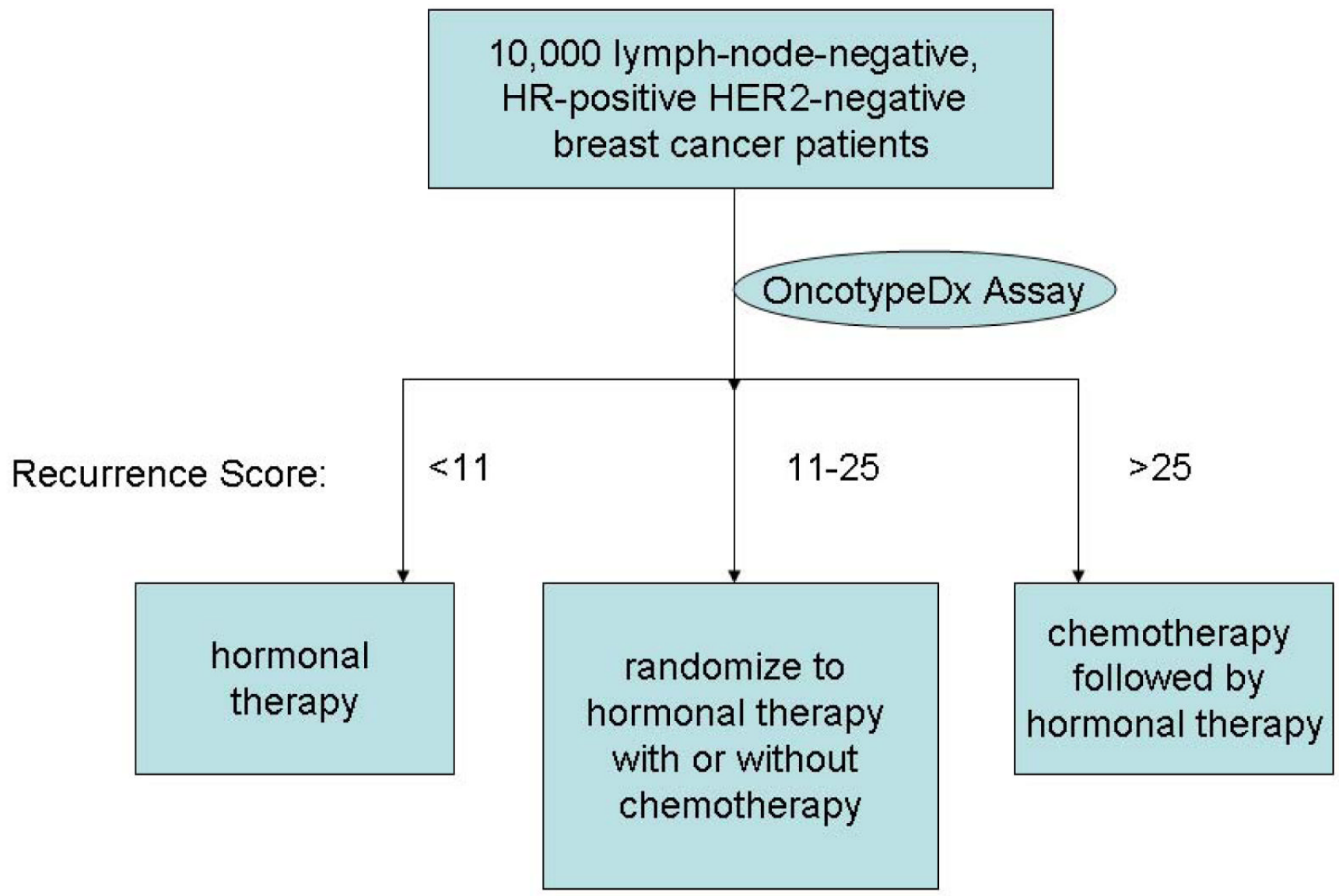

Figure 3.

TAILORx Treatment Schema (Modified from Sparano, 200726) 


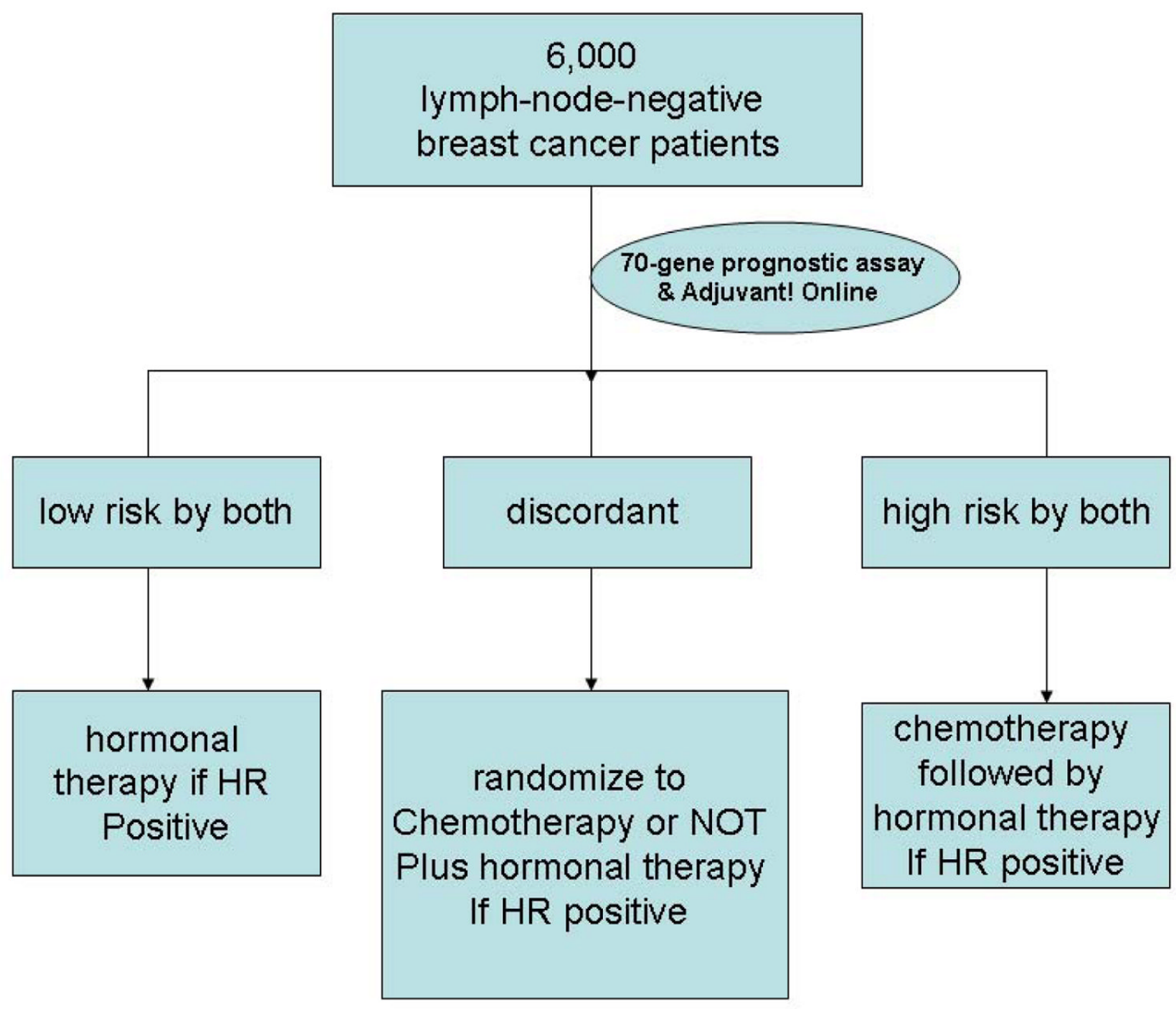

Figure 4.

MINDACT Trial Schema (Modified from MINDACT Trial outline ${ }^{27}$ )

Abbreviation: $\mathrm{HR}=$ hormone receptor 
Table 1

Tumor-Based Gene Expression Profiling Prognostic Models

\begin{tabular}{|c|c|c|}
\hline Test & Tissue Typ & Proposed Role \\
\hline $\begin{array}{l}\text { Mammaprint }{ }^{1} 10,11 \\
\text { (Amsterdam } 70 \text {-gene profile) }\end{array}$ & Fresh & $\begin{array}{l}\text { To predict risk of distant metastasis in node-negative breast cancer patients and to identify those patients } \\
\text { who would benefit from systemic chemotherapy }\end{array}$ \\
\hline $\begin{array}{l}\text { Oncotype Dx®13,17 } \\
\text { (21-gene recurrence score) }\end{array}$ & Fixed & $\begin{array}{l}\text { To identify women with node-negative, hormone-receptor-positive breast cancer who would benefit from } \\
\text { addition of chemotherapy to tamoxifen }\end{array}$ \\
\hline 76-gene prognostic signature ${ }^{18,19}$ & Fresh & $\begin{array}{l}\text { To predict disease free and overall survival in patients with node-negative early-stage breast cancer who } \\
\text { have not received systemic therapy }\end{array}$ \\
\hline Wound response ${ }^{20}$ & Fresh & To predict increased risk of metastasis and death in patients with breast cancer \\
\hline Two-gene ratio ${ }^{21}$ & Fixed & $\begin{array}{l}\text { To identify early stage steroid receptor positive breast cancer patients who would benefit from addition } \\
\text { of chemotherapy to tamoxifen }\end{array}$ \\
\hline Intrinsic subtype ${ }^{6,8}$ & Fresh & To predict clinical outcomes of breast cancer patients \\
\hline
\end{tabular}

